\title{
Correspondence
}

\section{Lubrication in hip joints}

SIR, We read with interest the article by Roberts et al. ${ }^{1}$ One possible explanation for the variance of their results with ours after trypsin and hyaluronidase digested synovial fluid on the frictional resistance of joints may be that they did not appear to deactivate the enzymes before performing the lubrication tests. These enzymes have well known effects on articular cartilage, and we think it likely that the authors would have obtained similar results to what we reported previously if they had taken the above precaution.

Department of

Orthopedic Surgery,

West Virginia University,

USA.

Shriners Burn Unit,

Massachusetts General Hospital,

Boston,

USA.

\section{Reference}

1 Roberts B J, Unsworth A, Mian N. Modes of lubrication in human hip joints. Ann Rheum Dis 1982; 41: 217-24.

SIR, We are grateful to Professors Radin and Swann for their interest in our paper and for their comments.

They are quite right to conclude that we did not deactivate the enzymes before performing the lubrication tests, but to avoid the possibility of any cartilage changes affecting the results we varied the order of testing the lubricants. This ensured that many of the tests using digested fluids were performed on the joints before those using control lubricants (undigested). If the existence of residual enzymes had affected the cartilage properties, then we would have expected the same changes to exist when the control fluid was used, since contact with the enzymes had already taken place. In fact closer examination of the results shows no consistent influence of the order of testing, and hence we can conclude that the effect of the enzymes on cartilage was not significant.

It still seems to us that the most likely cause of our discrepant results is that Professors Radin and Swann used constant heavy loading while we used the more physiological dynamic loading which is more likely to encourage fluid film lubrication.

Department of Engineering, University of Durham
A. UNSWORTH

B. J. ROBERTS N. MIAN

\section{Glycosylated haemoglobin in rheumatoid arthritis}

SIR, Differentiating between the anaemia of iron deficiency and that associated with diminished red cell production or shortened red cell survival is often difficult in patients with rheumatoid arthritis (RA). Bernstein et al have suggested that in normoglycaemic patients with RA glycosylated haemoglobin (GHb) levels in the normal range indicated bone marrow depression, which is generally the result of active disease. ${ }^{1}$.

We have tested this hypothesis in 9 consecutive patients admitted for investigation of anaemia. All were female. Their median age was 57 years (range 52-68), median haemoglobin (Hb) $8 \cdot 2 \mathrm{~g} / \mathrm{dl}$ (range $7 \cdot 2-10 \cdot 6$ ), and median erythrocyte sedimentation rate (ESR) $120 \mathrm{~mm}$ in first hour (range 53-140). All patients were normoglycaemic and 8 had active synovitis. A sternal bone marrow biopsy was performed and the aspirate stained for the presence of iron. GHb was estimated by affinity chromatography. ${ }^{2}$

Table $1 \mathrm{GHb}$ an $\mathrm{Hb}$ levels in anaemic patients with $\mathrm{RA}$. A: Iron staining present. B: Iron staining absent

\begin{tabular}{|c|c|c|c|}
\hline \multicolumn{2}{|l|}{$\mathbf{A}$} & \multicolumn{2}{|l|}{ B } \\
\hline$H b(g / d l)$ & $G H b(\%)$ & $H b(g / d l)$ & $G H b(\%)$ \\
\hline $10 \cdot 6$ & $5 \cdot 0$ & $7 \cdot 6$ & $6 \cdot 1$ \\
\hline $9 \cdot 0$ & $6 \cdot 3$ & $7 \cdot 2$ & 6.4 \\
\hline $8 \cdot 2$ & $6 \cdot 4$ & $7 \cdot 6$ & $6 \cdot 6$ \\
\hline $8 \cdot 7$ & $6 \cdot 5$ & $9 \cdot 6$ & $6 \cdot 7$ \\
\hline $7 \cdot 5$ & $6 \cdot 5$ & & \\
\hline
\end{tabular}

$\mathrm{GHb}$ levels in patients were significantly lower (median $6 \cdot 4$, range $5 \cdot 0-6 \cdot 7 \%$ ) than in normal controls (median $6 \cdot 9$, range $6 \cdot 2-7 \cdot 6 \%, p<0.001$ Mann-Whitney U test). In 5 patients the bone marrow aspirates stained normally for iron; in the other 4 there was no stainable iron. The GHb levels for the 2 groups are shown in Table 1. Three of 7 patients with $\mathrm{GHb}$ levels in the normal range $(>6.2 \%)$ had absent iron stores. One of 2 patients with low $\mathbf{G H b}$ levels had normal iron stores.

These results suggest that for individual patients $\mathrm{GHb}$ levels do not aid in the differentiation between iron deficiency anaemia and the anaemia associated with chronic disease.

Centre for Rheumatic Diseases, Glasgow University,

D. LEWIS

Glasgow G4 0EH

H. A. CAPELL

Department of Biochemstry,

University of Strathclyde,

D. K. TALWAR

Glasgow G4 0NR 